\title{
Fiber Optical Parametric Amplifier as Optical Signal Processor
}

\author{
Shunsuke Ono \\ Department of Electrical Engineering, Nara National College of Technology \\ Japan
}

\section{Introduction}

In this chapter, using numerical simulation and experimental results, the optical signal processing characteristics of Fiber-based Optical Parametric Amplifier (FOPA) using novel highly non-linear fibers (HNLFs) including Photonic Crystal Fibers (PCFs) will be described. In the last decade, to overcome the limitation of the electrical signal processing to the ultra high bit-rate optical data streams, ultrafast all-optical signal processing techniques including OTDM (Optical Time Division Multiplex), transparent wavelength conversion and alloptical sampling have been studied and proposed for over $160 \mathrm{~Gb} / \mathrm{s}$ optical data streams. For those ultrafast optical processing techniques, highly-nonlinear glass fiber-based optical signal processors have been promising due to its ultrafast response time of the nonlinear effects in glass fibers and low loss characteristic to the light signals.

FOPA exploiting four-wave mixing (FWM) occurs in HNLFs has been one of those highlynonlinear glass fiber-based all optical signal processors for ultrafast On-Off Keyed (OOK) optical signals such as Return-to-Zero (RZ) and Non-Return-to-Zero (NRZ). In recent years, with the emergences of novel glass fiber-based HNLFs with periodical microstructures including PCFs, which shows high nonlinearity using newly proposed germanium oxide silica glasses and novel heavy-metal oxide glasses, FWM-based FOPA has shortened its fiber device length drastically from tens of kilometers to tens of meters and improved its ultrafast optical signal processing characteristics remarkably. Also, with increasing application of the phase shift keyed (PSK) optical signals such as Differential Phase Shift Keying (DPSK) and Differential Quadrature Phase Shift Keying (DQPSK) in the feasibility studies of $40 \mathrm{~Gb} / \mathrm{s}-$ based optical communication systems, FWM-based FOPA has attracted much more attention again to its ultrafast optical phase controllability on time scale of a few femtoseconds. In Section 2 of this chapter, we will overview the development of the highly nonlinear glass fibers and the highly-nonlinear glass fiber-based optical signal processors including FWM-based- FOPAs. Numerical model of the signal processing characteristics of FWM-based FOPA will be introduced and overview the signal processing characteristics of FWM-based FOPA with the numerically simulated parametric gain characteristics of FWMbased FOPA In Section 4, we will introduce our experimental results of the parametric gain enhancement and the ultrafast signal processing experiments using highly-nonlinear silica glass-based FOPAs with over $160 \mathrm{~Gb} / \mathrm{s}$ ultrafast optical signal data streams. Practical 
applications of FOPA for optical signals are discussed and we will summarizes results and suggests directions of FOPA as an ultra fast optical signal processing device in Section 5.

\section{Optical Signal Processors based on Novel Glass-Based HNLFs}

\subsection{Fiber-Based Ultrafast Optical Signal Processors}

To overcome the limitation of the electrical signal processing to the ultra high bit-rate data streams, ultrafast all-optical signal processing techniques including the Optilcal Time Domain Multiplexing (OTDM), the transparent wavelength conversion and all-optical samplings have been eagerly studied and proposed in last decades (Westlund et al., 2002; Hedekvist et al., 1997; J. Li et al., 2001; Ho et al., 2001).

In particular, fiber-based optical signal processors exploiting the nonlinear optical effects in the glass fibers such as Self Phase Modulation (SPM), Cross Phase Modulation (XPM) and Four-Wave Mixing (FWM) and Stimulated Raman Scattering (SRS) have been promising to realize the ultrafast all optical processing devices (Optical Add/Drop Multiplexer (OADM), Demultiplexer (DEMUX), Wavelength Converter (WC), Fiber Optical Parametric Amplifier (FOPA) etc.) due to its ultrafast response time and low loss characteristic. 


\section{Signal}

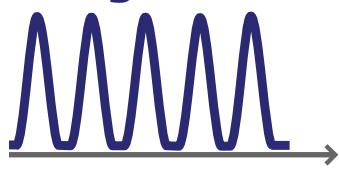

\section{DEMUX}

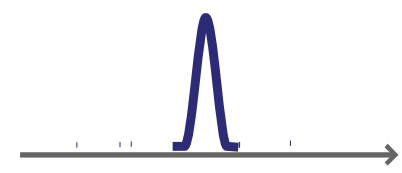

Signal

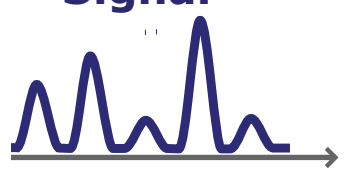

Signal

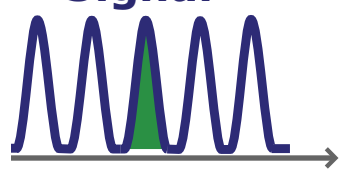

Dropping data (a)

\section{WC}

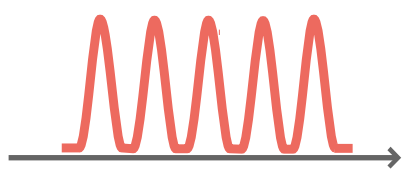

(b)

\section{DROP / ADD}

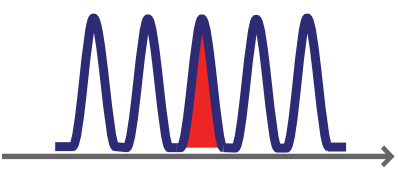

Adding data

(c)

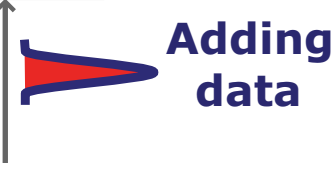

Fig. 1. Schematic diagrams of all optical ultrafast signal processors: (a) Optical DEMUX, (b) WC, and (c) OADM.

Figure 1 shows the schematic diagrams of all optical ultrafast signal processors: Optical DEMUX, WC and OADM. In particular, WC shows three active optical processings including wavelength conversion, re-amplification and re-shaping of distorted optical signals. The optical processing with re-amplification and re-shaping is usually called as optical " $2 \mathrm{R}^{\prime}$.

Generally, the nonlinear optical effects in glasses are known to occur on time scale of femto seconds ( $\mathrm{fs}$ ) which can realize the optical signal processing devices that have ultrafast response times. However, fiber-based optical signal processors have been also less realistic devices compared to other electrical signal processing devices because of their long fiber lengths. Because the fiber-based optical signal processors have been basically proposed using the silica-based glass fibers such as Dispersion Shifted Fibers (DSFs) without high nonlinearities to process the ultrafast optical signals, the fiber lengths of the silica-based nonlinear fiber devices have became very long in excess of a few hundred meters to achieve enough nonlinearities to process optical signals (Yang et al., 1997). 
In general, we can obtain larger nonlinear effects in glass fibers as the fiber lengths get longer. Therefore, in order to obtain high nonlinearities, long device lengths have been required for fiber-based optical signal processors. However, such long fiber lengths have led to the relative time delay among optical signals caused by the chromatic dispersions of the fibers. As in recent commercially deployed wavelength division multiplexing (WDM) optical telecommunication systems, equally frequency-spaced many optical signal channels have been used to enhance the transmission capacities of optical transmission systems. When the fiber lengths of the fiber-based optical signal processors become longer, the chromatic dispersions of these fiber devices give rise to the relative time delay among the optical signals in WDM transmission systems, which makes it difficult to process the ultrafast optical signals allocated in wide wavelength region simultaneously.

Thus, in order to work as ultrafast optical signal processors, undesirable long device lengths have been required for fiber-based optical devices to obtain enough high nonlinearities.

\subsection{Developments of Highly-Nonlinear Fibers based on Novel Glasses}

In last decade, due to the development of the synthetic techniques of the novel glass materials, nonlinear characteristics of glass materials have been improved drastically and made it realize to emerge various highly-nonlinear fibers (HNLFs).

In addition, owing to the establishment of the design techniques for the spatial strong photon localization in the periodically microstructured materials and fibers based on novel highly-nonlinear glasses, Photonic Crystal Fibers (PCFs) (Birks et al., 1997), have realized extremely high nonlinearities in considerably shorter fiber lengths than those of fibers with simple core-cladding structures. The emergences of these HNLFs and PCFs fabricated by novel highly-nonlinear glass materials have given different kinds of applications for ultrafast all optical processing devices. Among these optical signal processing applications in large capacity communication networks, wavelength conversion plays a major role in providing wavelength flexibility. Therefore, in particular, WC based on FWM have been mainly proposed as a possible application for newly proposed HNLFs and PCFs in order to evaluate the optical signal processing characteristics of these HNLFs and PCFs.

On the basis of the glass fiber compositions of HNLFs and PCFs, two types of highlynonlinear fibers have been proposed. One is the type of the glass ibers fabricated by conventional silica-based glasses and the other is the type of the glass fibers fabricated by non-silica-based glasses.

Table 1 shows the typical fiber parameters of various newly proposed silica-based and nonsilica-based highly-nonlinear fibers. In particular, heavy metal oxide glass-based highlynonlinear fibers are introduced as non-silica-based highly-nonlinear fibers.

As shown in Table 1, owing to the silica-based glass composition, silica-based highlynonlinear fiber shows very low loss $\alpha$ to be $0.0005 \mathrm{~dB} / \mathrm{m}$ (Onishi et al., 1997). This low loss characteristic of silica-based highly-nonlinear fiber means good compatibilities to the silicabased fibers used as transmission fibers in telecommunication systems, which leads to the low loss characteristics of silica-based highly-nonlinear fiber devices. In addition, nonlinear coefficient $\gamma$ of silica-based HNLF has been reported to be $\sim 20 \mathrm{~W}^{-1} \mathrm{~km}^{-1}$ (Onishi et al., 1997). Silica-based HNLF realizes the high refractive index contrast between the core and clad refractive indices using germanium-doped and fluoride-doped silica glasses as the materials for the core and clad. Silica-based PCF enhances nonlinearity due to its periodical special 
microstructure and achieves over three times higher nonlinear coefficient than that of the silica-based HNLF with simple core-cladding structure (Onishi et al., 1997).

Owing to the enhanced nonlinearities of silica-based HNLFs, the fiber lengths of optical signal processors using silica-based HNLFs have become much shorter than those of ones based on conventional DSFs. Also, with effectively shortening the required fiber length from kilometers to tens of meters, good optical signal processing characteristics have been evaluated for silica-based PCF using WC characteristic (Lee et al., 2003). Nevertheless, optical signal processing devices using silica-based HNLFs and PCFs still have tens of meters long fiber lengths. These rather long device lengths are much likely to bring the relative time delay among ultrafast optical signals over $160 \mathrm{~Gb} / \mathrm{s}$.

\begin{tabular}{|c|c|c|c|c|c|}
\hline $\begin{array}{c}\text { Glass } \\
\text { composition }\end{array}$ & Structure & $\begin{array}{c}n^{2} \\
\left(x 10^{-20} \mathrm{~m}^{2} / \mathrm{W}\right)\end{array}$ & $\begin{array}{c}\gamma \\
\left(\mathrm{W}^{-1} \mathrm{~km}^{-1}\right)\end{array}$ & $\begin{array}{c}D @ 1.5 \mu \mathrm{m} \\
(\mathrm{ps} / \mathrm{nm} / \mathrm{km})\end{array}$ & $\begin{array}{c}\alpha @ 1.5 \mu \mathrm{m} \\
(\mathrm{dB} / \mathrm{m})\end{array}$ \\
\hline $\begin{array}{l}\text { Germanium- } \\
\text { doped silica }\end{array}$ & Step index & $\sim 5$ & $\sim 20$ & - & 0.0005 \\
\hline Silica & PCF & 2.8 & 70 & -30 & 0.19 \\
\hline SF57 & PCF & 41 & 640 & $\sim 50$ & 2.6 \\
\hline Chalcogenide & Step index & 400 & - & - & $6.6 @ 1.3 \mu \mathrm{m}$ \\
\hline $\begin{array}{l}\text { Bsimuth } \\
\text { oxide }\end{array}$ & Step index & - & 1100 & -260 & 0.8 \\
\hline $\begin{array}{l}\text { Bsimuth } \\
\text { oxide }\end{array}$ & PCF & - & 580 & -9.9 & 1.9 \\
\hline
\end{tabular}

Table 1. Fiber parameters of silica glass-based and non-silica glass-based highly-nonlinear fibers.

As shown in Table 1, nonlinear coefficients of the heavy metal oxide glass-based HNLFs and PCFs become nearly over ten times larger than those of silica-based HNLFs and PCFs. These enhancements of nonlinearities of heavy metal oxide glass-based HNLFs and PCFs are due to the high refractive indices of heavy metal oxide glasses. Owing to the extremely high nonlinearities of heavy metal oxide glasses, heavy metal oxide glass-based HNLFs have shortened fiber device lengths successfully from tens of meters to a few meters. In addition, using bismuth oxide glass-based HNLF, less than 1 meter-long fiber-based WC has been proposed (Lee et al., 2005) However, bismuth oxide glass-based HNLF inevitably has large material dispersion similar with other non-silica-based HNLFs fabricated by heavy metal oxide glasses, which limits the working bandwidth of WC. In general, heavy metal oxide glass-based HNLFs have been found to have extremely high nonlinearities but at the same time suffer from large group velocity dispersions (GVD), large fiber losses and large splice losses between heavy metal oxide glass-based HNLFs and conventional silica-based 
fibers. In order to obtain broadband wavelength conversion characteristics using FWM, the glass fiber used as WC should have high nonlinearity, low dispersion and low loss characteristics (Inoue et al., 1992).

Thus, in order to realize practical optical signal processing devices such as OADM, WC, DEMUX and FOPA, high nonlinearity, low dispersion, low fiber loss, low coupling loss with conventional silica-based fiber are the required fiber characteristics for silica-glass based and heavy metal oxide glass-based highly nonlinear fibers. However, at the moment, we have not obtained the highly nonlinear fiber that can satisfy all required these fiber characteristics simultaneously.

\section{Optical Parametric Amplification using Four-Wave Mixing}

FWM has been as a main nonlinear effect to realize all optical signal processors. The optical signal processing characteristics of newly proposed HNLFs have been examined initially with the FWM efficiencies of WC using newly proposed HNLFs. Therefore, in this Section, we will introduce the precise theory of FWM and derive the coupling propagation equations of a signal, an idler and a pump light waves to establish numerical simulation model for FWM. In addition, we will overview the signal processing characteristics of FWM-based FOPA with the numerically simulated propagation characteristics of the signal along highlynonlinear glass fibers.

\subsection{Theory of Optical Parametric Amplification based on Four-Wave Mixing}

As cited in Section 1 and Section 2, FWM is one of the well-known nonlinear effects occur in glass fibers similar with SPM and XPM. SPM and XPM mainly affect the phase of the pulsed optical signals due to the additional nonlinear phase shifts and cause the spectral broadening of pulsed signals, whereas FWM gives rise to the energy exchange among optical signals. Figure 2 shows the schematic diagram of FWM in the frequency domain. As shown in Figure 2, when a signal light wave and two pump light waves are input into a nonlinear glass fiber, an additional new light wave that has higher frequency than those of the two pump light waves is generated. The additional new light wave is usually called as an "idler" light. 


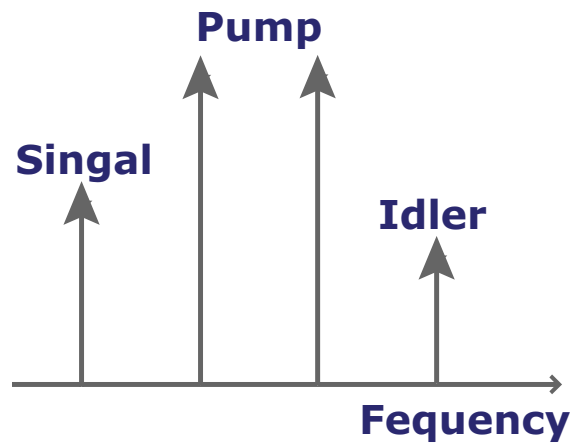

(a)

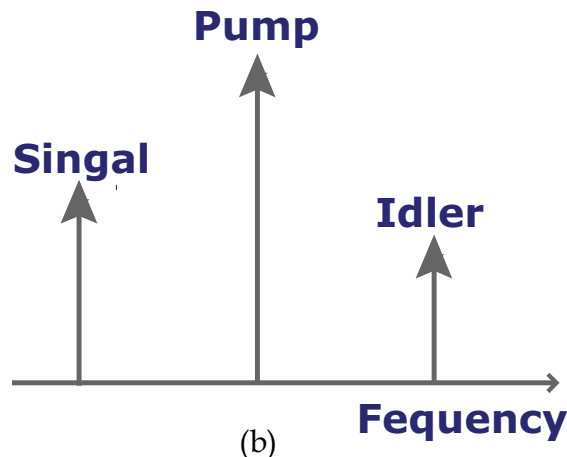

Fig. 2. Schematic diagrams of FWMs with (a) two pump lights and (b) one pump light in the frequency domain.

When the frequencies of two pump light waves are different, the relationship among the frequencies of the signal, the two pump and the idler light waves is given by,

$$
\omega_{\mathrm{i}}=\omega_{\mathrm{p} 1}+\omega_{\mathrm{p} 2}-\omega_{\mathrm{s}},
$$

where $\omega_{\mathrm{s}}, \omega_{\mathrm{p} 1}, \omega_{\mathrm{p} 2}$ and $\omega_{\mathrm{i}}$ are the frequencies of the signal, pumps and idler, respectively. As can be seen from Eq. (1), the energies of the signal and two pump lights are transferred to that of the idler. Therefore, FWM is usually thought to be the energy transfer phenomenon caused by the nonlinear effects in glass fibers.

When the frequencies of two pump lights are identical $\left(\omega_{\mathrm{p} 1}=\omega_{\mathrm{p} 2}=\omega_{\mathrm{p}}\right)$, the energies of two pump lights are equally transferred to those of the signal and the idler.

Therefore, Eq. (1) becomes as follows:

$$
2 \omega_{\mathrm{p}}=\omega_{\mathrm{s}}+\omega_{1} .
$$

This case where $\omega_{\text {pump1 }}$ and $\omega_{\text {pump2 }}$ coincide is called as "Degenerated" Four-Wave Mixing (DFWM). In this degenerated case, the frequencies of the three waves are symmetrically 
positioned relatively to each other in the frequency domain. Optical parametric amplification can be viewed from a quantum mechanical picture. Here, the degenerated parametric amplification case is manifested as the conversion of two pump photons at frequency $\omega_{\text {pump }}$ to a signal and an idler photon at frequencies $\omega_{\text {signal }}$ and $\omega_{\text {idler }}$. The conversion needs to satisfy the energy conservation relation as in Eq. (2). From the quantum-mechanical point of view, in the degenerated case, the photon momentum conservation relation is also needed to be satisfied as follows:

$$
\Delta \beta=\beta\left(\omega_{\mathrm{s}}\right)+\beta\left(\omega_{\mathrm{i}}\right)-2 \beta\left(\omega_{\mathrm{p}}\right)=0,
$$

where $\beta\left(\omega_{\mathrm{s}}\right), \beta\left(\omega_{\mathrm{i}}\right)$ and $\beta\left(\omega_{\mathrm{p}}\right)$ are the wave number in vacuum, respectively and $\Delta \beta$ is the mismatch among these wave numbers. For the rest of this chapter, we will focus on the degenerated case including one pump at $\omega_{\mathrm{p}}$, one signal at $\omega_{\mathrm{s}}$ and one idler light wave at $\omega_{\mathrm{i}}$. From the electromagnetic point of view, Eq. (3) is also called as "linear phase matching" condition because the wave numbers of the three light waves are directly linked to the phases of those light waves. Therefore, Eq. (3) can be considered to be the relative phase shift among the three light waves. The propagation equations of these three light waves in a transmission media are given by the following three nonlinear couple-mode equations (Agrawal., 1995):

$$
\begin{aligned}
& \frac{\mathrm{dA}_{\mathrm{p}}}{\mathrm{dz}}=\frac{1}{2} \alpha \mathrm{A}_{\mathrm{p}}+\mathrm{i} \gamma\left\{\left[\left|\mathrm{A}_{\mathrm{p}}\right|^{2}+2\left(\left|\mathrm{~A}_{\mathrm{s}}\right|^{2}+\left|\mathrm{A}_{\mathrm{i}}\right|^{2}\right)\right] \mathrm{A}_{\mathrm{p}}+2 \mathrm{~A}_{\mathrm{s}} \mathrm{A}_{\mathrm{i}} \mathrm{A}_{\mathrm{p}}^{*} \exp (\mathrm{i} \Delta \beta \mathrm{z})\right\}, \\
& \frac{\mathrm{dA}_{\mathrm{s}}}{\mathrm{dz}}=\frac{1}{2} \alpha \mathrm{A}_{\mathrm{s}}+\mathrm{i} \gamma\left\{\left[\left|\mathrm{A}_{\mathrm{s}}\right|^{2}+2\left(\left|\mathrm{~A}_{\mathrm{i}}\right|^{2}+\left|\mathrm{A}_{\mathrm{p}}\right|^{2}\right)\right] \mathrm{A}_{\mathrm{s}}+\mathrm{A}_{\mathrm{i}}^{*} \mathrm{~A}_{\mathrm{p}}^{2} \exp (-\mathrm{i} \Delta \beta \mathrm{z})\right\}, \\
& \frac{\mathrm{dA}_{\mathrm{i}}}{\mathrm{dz}}=\frac{1}{2} \alpha \mathrm{A}_{\mathrm{i}}+\mathrm{i} \gamma\left\{\left[\left|\mathrm{A}_{\mathrm{i}}\right|^{2}+2\left(\left|\mathrm{~A}_{\mathrm{s}}\right|^{2}+\left|\mathrm{A}_{\mathrm{i}}\right|^{2}\right)\right] \mathrm{A}_{\mathrm{p}}+\mathrm{A}_{\mathrm{s}}^{*} \mathrm{~A}_{\mathrm{p}}^{2} \exp (-\mathrm{i} \Delta \beta \mathrm{z})\right\} .
\end{aligned}
$$

where $A_{s} A_{i}$ and $A_{p}$ are the complex field amplitude of the signal, the idler and pump light waves, respectively. $\mathrm{z}$ is the longitudinal coordinate along the fiber as the transmission media. $\alpha$ is the loss of the glass fiber for these three light waves. $\gamma=2 \pi \mathrm{n}_{2} / \mathrm{A}_{\text {eff }}$ is the nonlinear coefficient of the fiber where $\mathrm{n}_{2}$ is the nonlinear parameter and $A_{\text {eff }}$ is the effective modal area of the fiber. In Eq. (4), Eq. (5) and Eq. (6), the first two terms are responsible for the nonlinear phase shift caused by SPM and XPM, respectively. The last terms of these couple-mode equations contribute to the energy transfer among the signal, the idler and the pump light waves.

Furthermore, to obtain further understanding to the propagation characteristics of the three light waves in the fiber, Eq. (4), Eq. (5) and Eq. (6) are rewritten by using $A_{j}=P_{j}^{1 / 2} \exp \left(i \phi_{j}\right)$ $(j=s, i, p)$ where $P_{j}(j=s, i, p)$ is the power of the three light waves and $\phi_{j}(j=s, i, p)$ is the phases of the these light waves, respectively. Thus, we can obtain practical propagating equations to describe the propagation characteristics of the signal, the idler and the pump lights as follows:

$$
\frac{\mathrm{dP}_{\mathrm{p}}}{\mathrm{dz}}=\alpha \mathrm{P}_{\mathrm{p}}-4 \gamma\left[\left(\mathrm{P}_{\mathrm{s}} \mathrm{P}_{\mathrm{i}} \mathrm{P}_{\mathrm{p}}^{2}\right)^{1 / 2} \sin \theta\right]
$$




$$
\begin{gathered}
\frac{\mathrm{dP}_{\mathrm{s}}}{\mathrm{dz}}=\alpha \mathrm{P}_{\mathrm{s}}+2 \gamma\left[\left(\mathrm{P}_{\mathrm{s}} \mathrm{P}_{\mathrm{i}} \mathrm{P}_{\mathrm{p}}^{2}\right)^{1 / 2} \sin \theta\right], \\
\frac{d \mathrm{P}_{\mathrm{i}}}{\mathrm{dz}}=\alpha \mathrm{P}_{\mathrm{i}}+2 \gamma\left[\left(\mathrm{P}_{\mathrm{s}} \mathrm{P}_{\mathrm{i}} \mathrm{P}_{\mathrm{p}}^{2}\right)^{1 / 2} \sin \theta\right], \\
\frac{\mathrm{d} \theta}{\mathrm{dz}}=\Delta \beta+\gamma\left(2 \mathrm{P}_{\mathrm{p}}-\mathrm{P}_{\mathrm{s}}-\mathrm{P}_{\mathrm{i}}\right)+\gamma\left[\left(\mathrm{P}_{\mathrm{P}}^{2} \mathrm{P}_{\mathrm{i}} / \mathrm{P}_{\mathrm{s}}\right)^{1 / 2}+\left(\mathrm{P}_{\mathrm{P}}^{2} \mathrm{P}_{\mathrm{s}} / \mathrm{P}_{\mathrm{i}}\right)^{1 / 2}-4\left(\mathrm{P}_{\mathrm{s}} \mathrm{P}_{\mathrm{i}}\right)^{1 / 2}\right] \cos \theta .
\end{gathered}
$$

Here, Eq. 10 describes the propagation characteristics of the relative phase among three light waves. The first term of Eq. 10 is the contribution from the linear phase shift that does not depend on the powers of three light waves. The second and third term of Eq. 10 show the contributions from the nonlinear phase shifts caused by the nonlinearity of the glass fiber.

To simulate the frequency dependence of the optical amplification characteristics based on FWM, we will expand the linear phase shift $\Delta \beta$ in Taylor series to the fourth order around the zero-dispersion frequency $\omega_{0}$. Thus, we can obtain the frequency-dependent linear phase shift $\Delta \beta$ given by,

$$
\Delta \beta=\left\{\beta_{3}\left(\omega_{\mathrm{p}}-\omega_{0}\right)+\frac{\beta_{4}}{2}\left[\left(\omega_{\mathrm{p}}-\omega_{0}\right)^{2}+\frac{1}{6}\left(\omega_{\mathrm{p}}-\omega_{\mathrm{s}}\right)^{2}\right]\right\}\left(\omega_{\mathrm{p}}-\omega_{\mathrm{s}}\right)^{2} .
$$

Here, $\beta_{3}$ and $\beta_{4}$ is the third and fourth order dispersions defined at $\omega_{0}$. Also, $\beta_{2}$ (the second order dispersion of $\beta(\omega))$ becomes zero because $\beta_{2}\left(\omega_{0}\right)=0$. Usually, $\beta_{2}$ and $\beta_{3}$ are called as "dispersion" and "dispersion slope" of the fiber, respectively. In addition, when the pump frequency $\omega_{\mathrm{p}}$ is chosen to become $\omega_{\mathrm{p}}=\omega_{0}$, the frequency dependent part of $\Delta \beta$ is fully dominated by the fourth-order dispersion $\beta_{4}$. Therefore, the gain bandwidth of FOPA is strongly limited by the fourth-order dispersion $\beta_{4}$ (Marhic et al., 1996). To realize the broad band optical signal wavelength converter using FOPA based on FWM, the value of $\beta_{4}$ has been improved in silica-based highly nonlinear glass fibers (Hirano et al., 2005). Higher order dispersions become an important limiting factor as the optical signal operating bandwidth $\Delta \lambda=\left|\lambda_{\mathrm{p}}-\lambda_{\mathrm{s}}\right|$ exceeds $100 \mathrm{~nm}$.

\subsection{Numerical Analysis on Gain Characteristics of FWM-based FOPA}

Next, using the simulation model based on the reduced propagation equations (7), (8), (9) \& (10), we will overview the signal processing characteristics of FWM-based FOPA with the numerically simulated parametric gain characteristics of FWM-based FOPA. In this numerical simulation, we used further reduced Eq. (12) and Eq. (13) obtained by neglecting $\beta_{4}$ and by assuming that FOPA are operated in a phase-matching condition that the relative phase remains near $\pi / 2$ in Eq. (10) and Eq. (11). Eq. (12) and Eq. (13) are given by;

$$
\begin{gathered}
\kappa=\Delta \beta+2 \gamma\left(\mathrm{P}_{\mathrm{p}}-\mathrm{P}_{\mathrm{s}}-\mathrm{P}_{\mathrm{i}}\right), \\
\Delta \beta=-\frac{2 \pi \mathrm{c} \lambda_{0}^{3}}{\lambda_{\mathrm{P}}^{3} \lambda_{\mathrm{S}}^{2}} \frac{\mathrm{dD}_{\mathrm{C}}}{\mathrm{d} \lambda}\left(\lambda_{\mathrm{P}}-\lambda_{\mathrm{S}}\right)^{2}\left(\lambda_{\mathrm{P}}-\lambda_{0}\right),
\end{gathered}
$$

where $\kappa$ and $\Delta \beta$ show the linear and total phase mismatch parameters, respectively. $\gamma$ is the nonlinear coefficient of HNLF. $P_{j}(j=s, p, i)$ and $\lambda_{j}(j=s, p, i)$ show the powers and wavelengths of signal, pump and idler, respectively. $\mathrm{dD} / \mathrm{d} \lambda_{\mathrm{c}}$ is the dispersion slope at the 
zero dispersion wavelength of HNLF. It is known that the parametric gain of FWM-based FOPA shows the maximum value when FWM-based FOPA is operated in the total phasematching condition $\kappa=0$ is satisfied.

As shown in Eq. (12), the total phase mismatch consists of the linear phase mismatch part $\Delta \beta$ and the nonlinear phase mismatch part $2 \gamma\left(\mathrm{P}_{\mathrm{p}}-\mathrm{P}_{\mathrm{s}}-\mathrm{P}_{\mathrm{i}}\right)$. The linear phase mismatch part

$\Delta \beta$ does not depend on the powers of signal, pump and idler. Therefore, $\Delta \beta$ becomes the constant phase mismatch factor for FOPA. On the other hand, the nonlinear phase mismatch part $2 \gamma\left(\mathrm{P}_{\mathrm{p}}-\mathrm{P}_{\mathrm{s}}-\mathrm{P}_{\mathrm{i}}\right)$ apparently depends on the powers of signal, pump and idler.

Therefore, when the powers of signal, pump and idler are varied in FWM-based FOPA, parametric gain characteristics of FWM-based FOPA are affected sensitively by the total phase mismatching due to the variation of these powers.

Firstly, we will overview this parametric gain variation of FWM-based FOPA induced by the input power with the gain characteristics of FWM-based FOPA with different pump powers shown in Figure 3.

Table 2 shows the fiber parameters of silica-based HNLF used in this analysis on the parametric gain characteristics of FWM-based FOPA. In this numerical simulation, the parametric gain media of FOPA is assumed to be a germanium-doped silica-based HNLF with nonlinear coefficient $25 \mathrm{~W}^{-1} \mathrm{Km}^{-1}$ and $150 \mathrm{~m}$ of the fiber length. Then, the pump wavelength is set to be $1562 \mathrm{~nm}$ longer than zero dispersion wavelength of the fiber 1555.5 $\mathrm{nm}$. The input signal power into HNLF is $-30 \mathrm{dBm}$. As shown in Figure 3, the parametric gain of FOPA becomes to show two symmetrical peaks about the pump wavelength as the higher pump power input into HNLF. At the wavelengths where the parametric gain shows its peaks, total phase-matching condition $\kappa=0$ is fully satisfied. Whereas the parametric gain shows peaked gain shape with high pump power $\left(\mathrm{P}_{\mathrm{p}}=30 \mathrm{dBm}\right)$, almost flat parametric gain shape is obtained with low pump power $\left(P_{p}=20 \mathrm{dBm}\right)$. This pump power dependence of the parametric gain characteristics shows that the difference in the parametric gain evolution rates is induced by the power-dependent nonlinear phase mismatch part $2 \gamma\left(\mathrm{P}_{\mathrm{p}}-\mathrm{P}_{\mathrm{s}}-\mathrm{P}_{\mathrm{i}}\right)$. Generally, it is known that the parametric gain characteristics are exponentially proportional to the applied pump power in the wavelength region where the perfect phase-matching condition $\kappa=0$ is satisfied. Also, the parametric gain characteristics are quadratically proportional to the applied pump power in the wavelength region where the linear phase-matching condition $\Delta \beta=0$ is satisfied. Figure 4 shows the simulated parametric gain evolutions of a signal and an idler along the silica-based HNLF with two different wavelengths. One is $1540 \mathrm{~nm}$ where the total phase-matching condition $\kappa=0$ is found to be almost satisfied show in Figure 3. The other is $1560.5 \mathrm{~nm}$ near the pump wavelength $1562 \mathrm{~nm}$ where the linear phase-matching condition $\Delta \beta=0$ is satisfied.

As shown in Figure 4, the difference between parametric gains at $1540 \mathrm{~nm}$ and $1560.5 \mathrm{~nm}$ becomes larger as the signal propagation distance gets longer. This implies that the fiber length should be shortened to obtain the flat parametric gain shape of FOPA. On the other hand, long fiber length is required to obtain large parametric gain of FOPA. Thus, it is difficult to realize large and flat gain characteristics of FOPA simultaneously. So far, in order to achieve large and flat gain characteristics of FOPA, the gain enhancement schemes of FOPA with broad gain characteristics have been proposed. Therefore, in Section 4, in order to realize the FOPA with large and flat gain characteristics, we will propose a novel parametric gain enhancement scheme of FOPA with flat gain characteristics. 


\begin{tabular}{||cc||}
\hline \multicolumn{2}{|c||}{ Simulation parameters of HNLF } \\
\hline Glass composition & Germanium $\mathrm{SiO}_{2}$ \\
Fiber length $[\mathrm{m}]$ & 150 \\
Nonlinear coefficient $\left[\mathrm{W}^{-1} \mathrm{~km}^{-1}\right]$ & 25 \\
Zero dispersion wavelength $[\mathrm{nm}]$ & 1555.5 \\
Dispersion Slope $\left[\mathrm{ps} / \mathrm{nm} / \mathrm{km}^{2}\right]$ & 0.03 \\
Fiber loss $[\mathrm{dB} / \mathrm{km}]$ & 0.5 \\
\hline \hline
\end{tabular}

Table 2. Fiber parameters of silica-based HNLF.

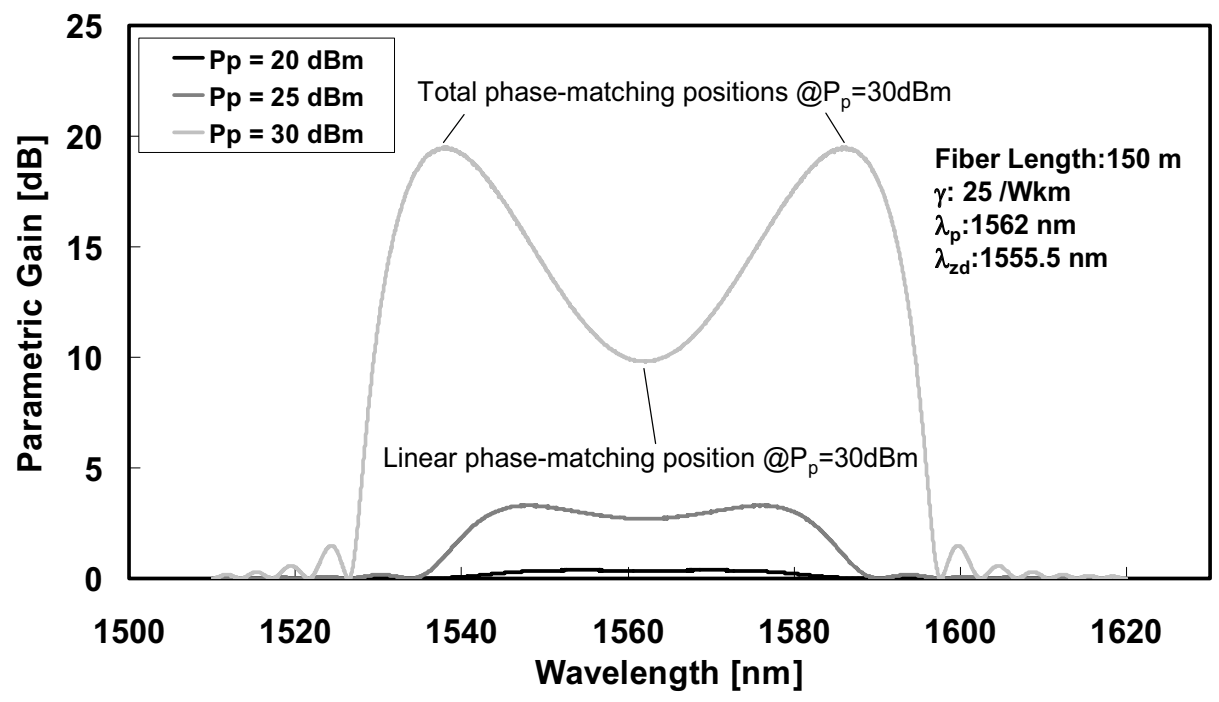

Fig. 3. Simulated parametric gain characteristics of silica glass-based FOPA with various pump powers $\left(\mathrm{P}_{\mathrm{p}}=10 \mathrm{dBm}, 20 \mathrm{dBm}\right.$ and $\left.30 \mathrm{dBm}\right)$. 


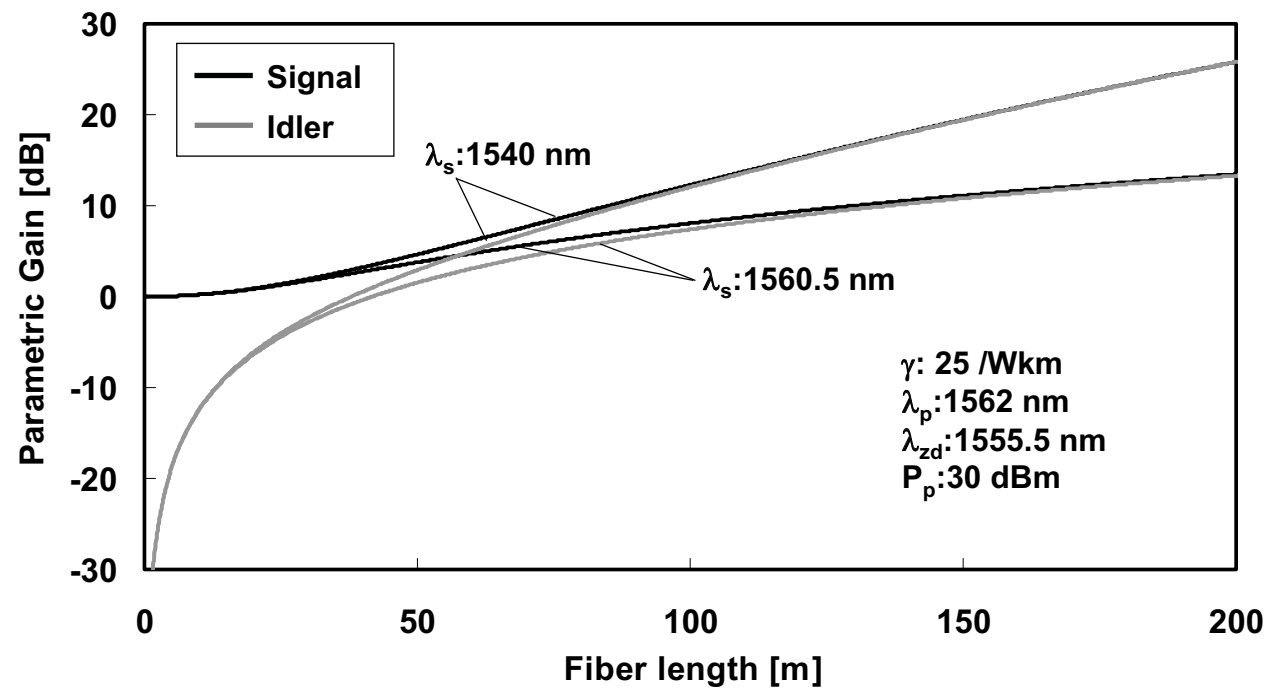

Fig. 4. Fiber propagation characteristics of the parametric gains of signals and idlers with different wavelengths $\left(\lambda_{s}=1540 \mathrm{~nm}\right.$ and $\left.1560.5 \mathrm{~nm}\right)$.

\section{Application of FOPA to Ultrafast Optical Signal Processing Device}

In this Section, we will introduce our experimental results of the ultrafast signal processing experiments using single-pumped FOPAs as an broadband ultrafast optical DEMUX with large parametric gain characteristics for over $160 \mathrm{~Gb} / \mathrm{s}$ optical signal data streams. On the basis of our proposed design schemes for FOPA, we performed evaluations on the parametric gain characteristics of the single-pumped cascaded silica-glass-based HNLFs with different dispersions and dispersion slopes.

As results of this evaluation, it was shown that over $20 \mathrm{~dB}$ parametric gains can be achieved in $\sim 50 \mathrm{~nm}$ band width in C-band wavelength region. Apparent improvement in the parametric gain characteristics was observed in the wavelength region near the pump wavelength. Furthermore, to verify the capability of our proposed FOPA as an ultrafast optical signal processor, we performed transparent $160 \mathrm{~Gb} / \mathrm{s}$ - $10 \mathrm{~Gb} / \mathrm{s}$ DEMUX experiments using $160 \mathrm{~Gb} / \mathrm{s}$ On-Off keyed (OOK) data using the parametric gain-enhanced FOPA by our proposed design scheme. As a result, no error penalty occurred for $160 \mathrm{~Gb} / \mathrm{s}$ ultrafast data streams in the transparent $160 \mathrm{~Gb} / \mathrm{s}-10 \mathrm{~Gb} / \mathrm{s}$ DEMUX experiment.

\subsection{Broadband FOPA Design Scheme with Perfect Phase-matching Condition}

So far, FOPA and Nonlinear Loop Mirror (NOLM) have been proposed as all optical fiberbased DEMUX. In principle, the optical gating action controlled by optical clock pulses is required for optical DEMUX. Figure 5 shows the comparison of the optical DEMUX characteristics of NOLM-based DEMUX and FOPA-based DEMUX.

As shown in Figure 5, NOLM-based DEMUX cannot amplify the input signal and can bring the interference between incoming and counter propagate pulse. For ultrafast optical fast 
signals, this interference between incoming and counter propagate pulse can deteriorate the optical data streams in NOLM-based DEMUX. On the other hand, FOPA based DEMUX can amplify the incoming data signal with parametric gain. Also, there is no interference of data pulses in FOPA-based DEMUX. Therefore, in this work, we used FOPA as optical DEMUX for ultrafast optical signal processing. The results of transparent $160 \mathrm{~Gb} / \mathrm{s}-10 \mathrm{~Gb} / \mathrm{s}$ DEMUX experiments using FOPA-based ultrafast DEMUX are will be shown in Section 4.

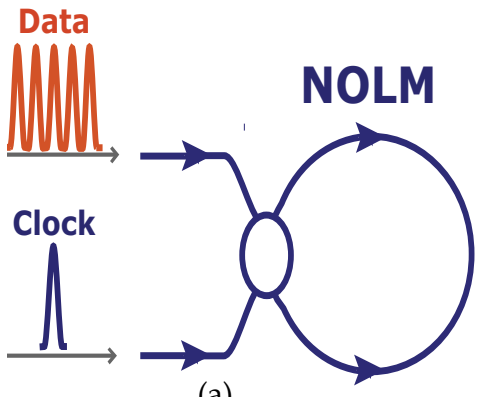

(a)

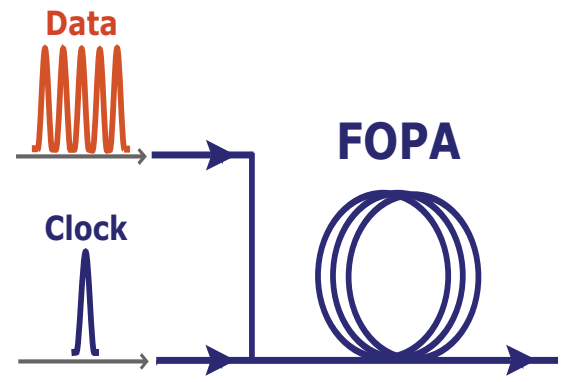

(b)

Fig. 5. Schematic diagrams of the principles of the optical gating operations in (a) NOLM and (b) FOPA.

In order to realize the broadband ultrafast optical DEMUX using FOPA, large FOPA gain characteristics in a wide wavelength region should be realized. So far, in order to achieve large and broad gain characteristics of FOPA, the gain enhancement schemes of FOPA with broad gain characteristics have been proposed using many cascaded HNLFs with dual pump scheme. However, using many HNLFs and dual pump scheme is not suitable way to realize the practical FOPA for optical signal processors because of the complicated HNLF's configuration and the use of two pump lasers, which leads to the increase of splice losses between HNLFs and the less cost effectiveness. Particularly, in order to realize an optical DEMUX using FOPA, the optical gating of the optical DEMUX should be controlled by single pump light source as an optical clock pulse because it is extremely difficult to adjust and control the relative timing among ultrafast optical signals over $160 \mathrm{~Gb} / \mathrm{s}$ and multipump light pulses. In order to realize a practical FOPA with large and broad parametric gain characteristics using single pump light source, simpler design scheme of FOPA are required. Therefore, we propose a simple and practical design scheme to achieve large and broad parametric gain characteristics of FOPA exploiting the perfect phase-matching condition of FWM.

Figure 6 shows the optical parametric gain characteristics of FOPA achieved in the perfectmatched and linear phase-matching conditions. When the perfect phase-matching condition $\kappa=\Delta \beta+2 \gamma\left(\mathrm{P}_{\mathrm{p}}-\mathrm{P}_{\mathrm{s}}-\mathrm{P}_{\mathrm{i}}\right)=0$ is achieved, the parametric gain shows the exponential characteristics in terms of the pump power as shown in Section 3. In addition, if the linear phase-matching condition $\Delta \beta=0$ is only satisfied, the parametric gain characteristics becomes quadratic one. In our design scheme, we particularly tried to improve the gain characteristics in the wavelength region near the pump wavelength because the gain depletion occurs significantly in that wavelength region. 
Figure 7 shows the schematic design concept of the switching gain band width enhancement using quasi-cascaded two HNLFs with different dispersions and dispersion slopes. The term "quasi" is used because there is not much difference between the optical parameters of these two HNLFs. In this two HNLFs configuration scheme, the zero dispersion wavelength of $1^{\text {st }}$ HNLF is set to become slightly shorter than the pump wavelength, which makes the wavelength of the parametric gain peak relatively far from the pump wavelength. Also, the zero dispersion wavelength of $2^{\text {nd }}$ HNLF is set to become shorter than that of the $1^{\text {st }}$ HNLF in order to make the wavelength of the gain peak of $2^{\text {nd }}$ HNLF longer. In addition, the dispersion slope of the $2^{\text {nd }}$ HNLF is set to be relatively steeper than that of $1^{\text {st }}$ HNLF to make the maximum gain peak of the $2^{\text {nd }}$ HNLF shift toward the pump wavelength in the shorter wavelength. With this design scheme, the improvement of the parametric gain characteristics particularly in the wavelength region near the pump wavelength can be expected.

\subsection{Experimental Setup}

\subsubsection{Evaluations of Optical Parametric Gain Characteristics of FOPA}

Figure 8 shows the experimental configuration for the parametric gain characteristic evaluation. The proposed design scheme for HNLFs to achieve high and broadened parametric switching gains was tested using a quasi-cascaded HNLF consists of two HNLFs.

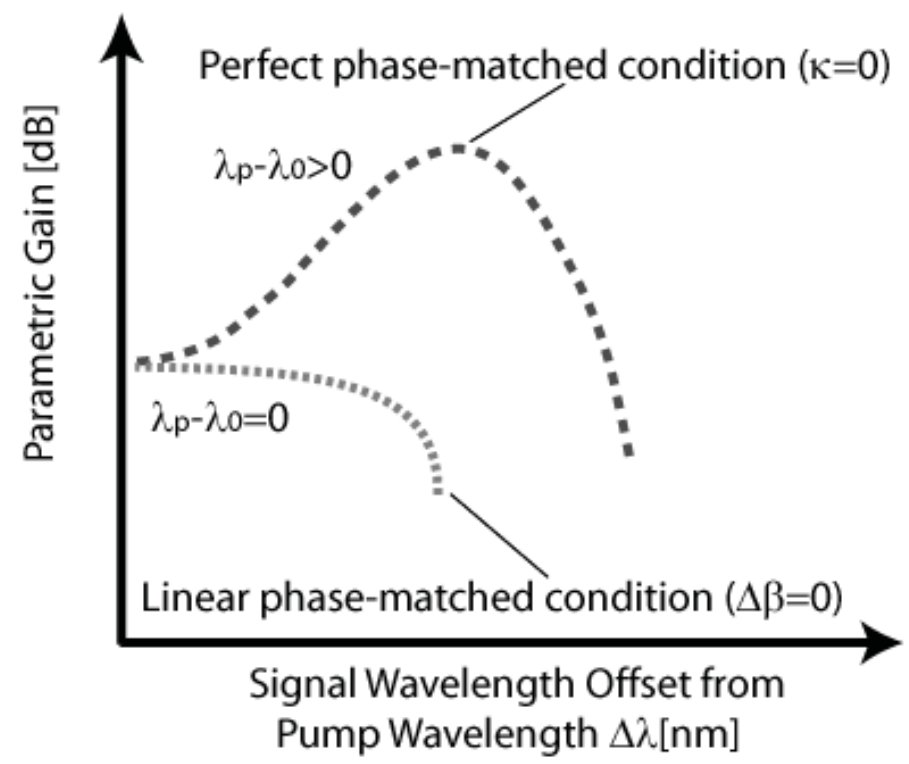

Fig. 6. Schematic diagrams of the optical parametric gain characteristics of FOPA achieved in the perfect-matched $(\kappa=0)$ and the linear phase-matching conditions $(\Delta \beta=0)$. 


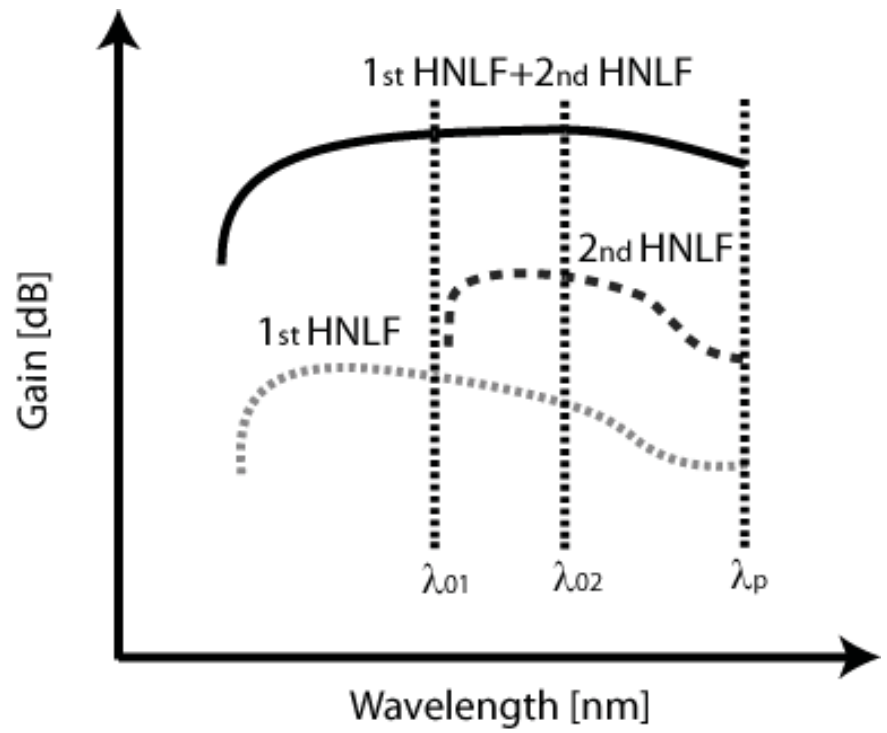

Fig. 7. Schematic diagram of the design concept for the parametric gain bandwidth enhancement using the quasi-cascaded two HNLFs with different dispersions and dispersion slopes.

Two HNLFs have the zero dispersion slopes and the zero dispersion wavelengths $\left(\lambda_{01}\right.$, $\left.\mathrm{dD} / \mathrm{d} \lambda_{01}\right)=\left(1570 \mathrm{~nm}, 0.03 \mathrm{ps} / \mathrm{nm}^{2} / \mathrm{km}\right)$ and $\left(\lambda_{02}, \mathrm{dD} / \mathrm{d} \lambda_{2}\right)=\left(1555 \mathrm{~nm}, 0.035 \mathrm{ps} / \mathrm{nm}^{2} / \mathrm{km}\right)$, respectively. The nonlinear coefficients $\gamma$ of the HNLFs are $\sim 21 \mathrm{~W}^{-1} \mathrm{~km}^{-1}$. A modelocked Fiber laser (MLFL) as a signal source generated a $10 \mathrm{GHz}$ optical pulse train at wavelengths in the wavelength region from $1520 \mathrm{~nm}$ to $1550 \mathrm{~nm}$. A tunable laser source was used as pump source, which was modulated by an Electro-Absorbtive (EA) modulator and a $\mathrm{LiNbO}_{3}$ intensity modulator (LN, $10 \mathrm{~Gb} / \mathrm{s}$, PRBS: ${ }^{23}-1$ ) to realize the wide pulse width with the repetition rate $311 \mathrm{MHz}$ and a high peak power at the fiber input. Maximum pump peak power was about $43 \mathrm{dBm}$. A signal was input to the quasi-cascaded HNLFs together with a pump pulse that had $\sim 45$ degree-aligned polarization to that of the signal pulse. The wavelength of the pump pulse was $1570.5 \mathrm{~nm}$ to have almost the same wavelength of the $1^{\text {st }}$ HNLF. The pulse widths (FWHM) of the signal and control pulses were 1.6 ps and 25 ps, respectively. 


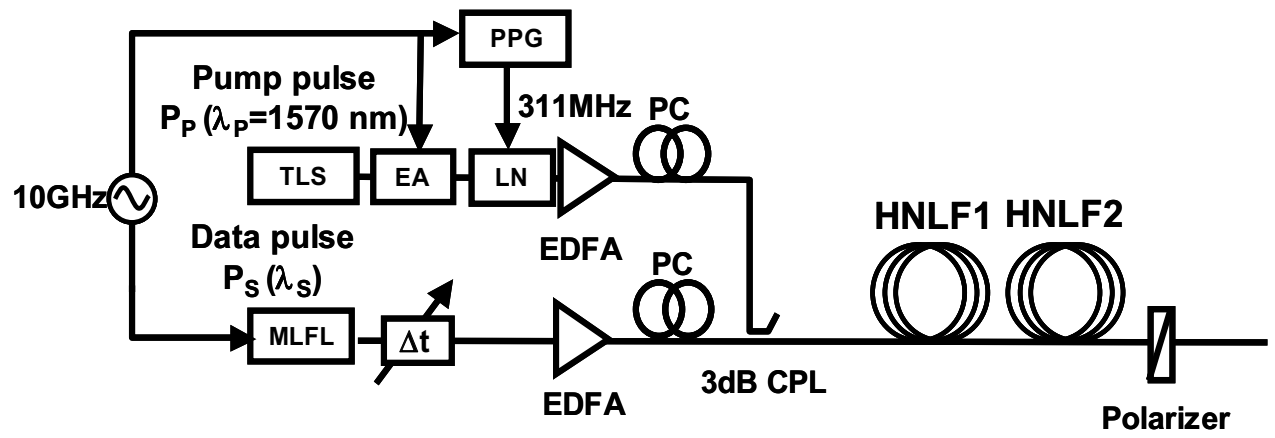

Fig. 8. Schematic diagram of the experimental setup for the evaluation on the parametric gain characteristic of proposed quasi-cascaded HNLFs.

\subsubsection{Gb/s -10 Gb/s DEXUM Experiment using Quasi-Cascaded HNLFs}

Furthermore, we evaluated the signal processing characteristics of the perfect phasematching FOPA using ultrafast pulse trains. $160 \mathrm{~Gb} / \mathrm{s}$ - $10 \mathrm{~Gb} / \mathrm{s}$ OTDM DEMUX experiment was performed using another modelocked fiber laser, which generates $1.6 \mathrm{ps}$ light pulse with the pulse repetition rate $10 \mathrm{GH} / \mathrm{z}$ at the wavelength $\lambda_{\mathrm{s}}=\sim 1540 \mathrm{~nm}$ where the parametric gain characteristics improved by our proposed FOPA design scheme. This $10 \mathrm{~Gb} / \mathrm{s}$ pulse was optically time-division multiplexed to generate a single polarized 160 $\mathrm{Gb} / \mathrm{s}$ data pulse train. Using this pulse train as a signal, we performed $160 \mathrm{~Gb} / \mathrm{s}-10 \mathrm{~Gb} / \mathrm{s}$ OTDM DEMUX experiments to verify the capability of the proposed FOPA as an ultrafast optical signal processor.

\subsection{Results}

\subsubsection{Parametric Gain Characteristic of Quasi-Cascaded HNLFs}

Figure 9 shows the parametric signal gain characteristics of $1^{\text {st }}, 2^{\text {nd }}$ and $1^{\text {st }}+2^{\text {nd }}$ HNLFs. As shown in Figure 9, parametric signal gain characteristics of $1^{\text {st }}$ and $2^{\text {nd }}$ HNLF show the parametric gain peaks at the wavelengths $\sim 1520 \mathrm{~nm}$ and $\sim 1550 \mathrm{~nm}$ where phase-matching conditions $\kappa=0$ in $1^{\text {st }}$ and $2^{\text {nd }}$ HNLF are satisfied. Parametric signal gain characteristic of quasi-cascaded HNLFs (1st $+2^{\text {nd }}$ HNLFs) is also shown in Figure 8. As shown in Figure 8, with our proposed design concept for FOPA, parametric signal gain characteristic of cascaded HNLFs becomes flat shape compared to those of $1^{\text {st }}$ and $2^{\text {nd }}$ HNLF. As results of this evaluation, it was shown that over $\sim 20 \mathrm{~dB}$ parametric gains can be achieved in $\sim 50 \mathrm{~nm}$ bandwidth (from $1510 \mathrm{~nm}$ to $1560 \mathrm{~nm}$ ) in C-band wavelength region. In particular, improvement in the parametric gain characteristics was observed in the wavelength region near the pump wavelength $1570 \mathrm{~nm}$. 


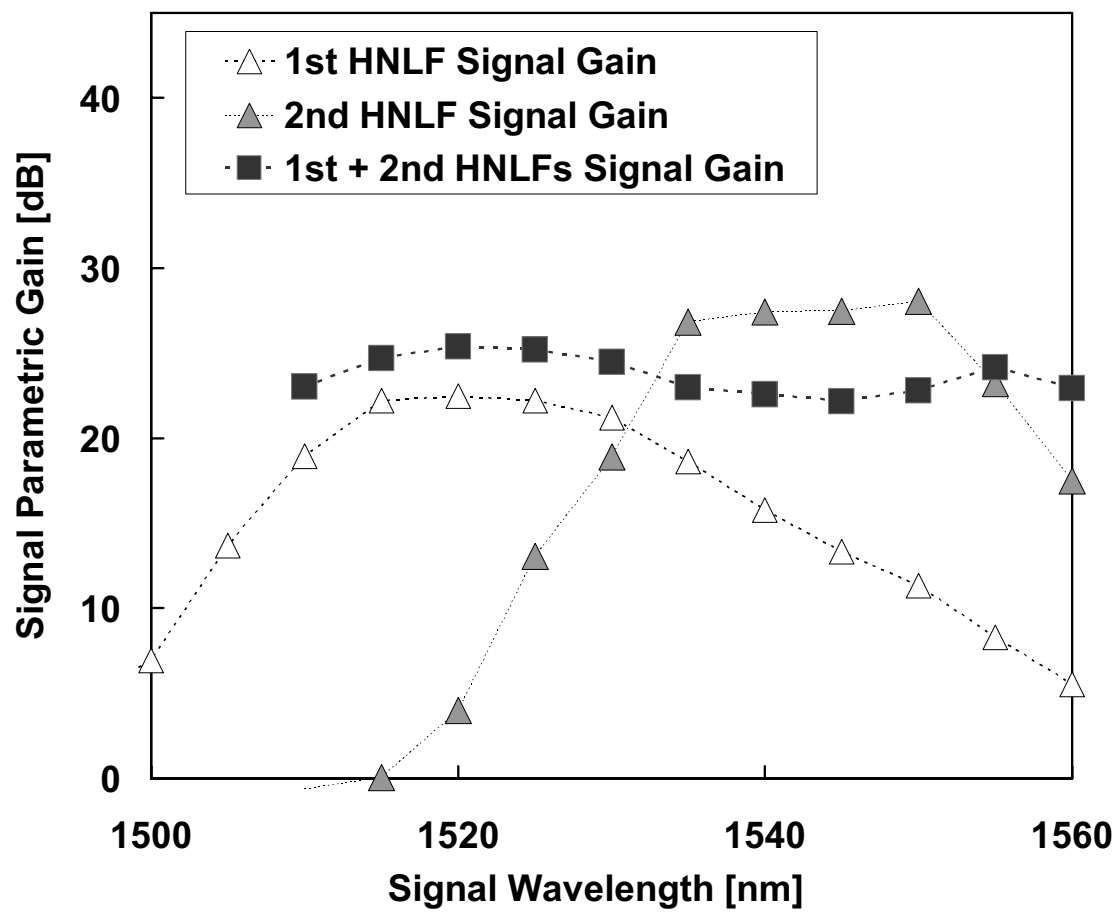

Fig. 9. Parametric gain characteristics of $1^{\text {st }} \mathrm{HNLF}, 2^{\text {nd }} \mathrm{HNLF}$ and $1^{\text {st }}+2^{\text {nd }} \mathrm{HNLFs}$.

\subsubsection{High Speed Switching Characteristics in $160 \mathrm{~Gb} / \mathrm{s}-10 \mathrm{~Gb} / \mathrm{s}$ DEMUX}

Figure 10 shows the measured bit error rates (BERs) obtained by $160 \mathrm{~Gb} / \mathrm{s}-10 \mathrm{~Gb} / \mathrm{s}$ OTDM DEMUX experiments. The eye-patterns in the BER measurements of $10 \mathrm{~Gb} / \mathrm{s}$ reference and $160 \mathrm{~Gb} / \mathrm{s}-10 \mathrm{~Gb} / \mathrm{s}$ OTDM DEMUX are also shown (inset (a) and inset (b)). As shown in Figure 10, error free operations were demonstrated successfully without wavelength conversion for the OTDM DEMUXs of $160 \mathrm{~Gb} / \mathrm{s}$ pulse trains. No penalty was occurred in the $160 \mathrm{~Gb} / \mathrm{s}-10 \mathrm{~Gb} / \mathrm{s}$ transparent OTDM experiment compared to the $10 \mathrm{~Gb} / \mathrm{s}$ reference BER curve. Therefore, No degradations of demuxed $10 \mathrm{~Gb} / \mathrm{s}$ signal pulses occur after optical signal processing using our proposed FOPA-based optical demultiplexer. Also, compared to the input $160 \mathrm{~Gb} / \mathrm{s}$ pulse trains, DEMUXed $10 \mathrm{~Gb} / \mathrm{s}$ signal pulses show over 20 $\mathrm{dB}$ pulse gain induced by a perfect phase-matching. As results of $160 \mathrm{~Gb} / \mathrm{s}-10 \mathrm{~Gb} / \mathrm{s}$ OTDM DEMUX experiments, our proposed FOPA is considered to be work as optical DEMUX with large parametric gain for up to $160 \mathrm{~Gb} / \mathrm{s}$ ultrafast optical data streams. 


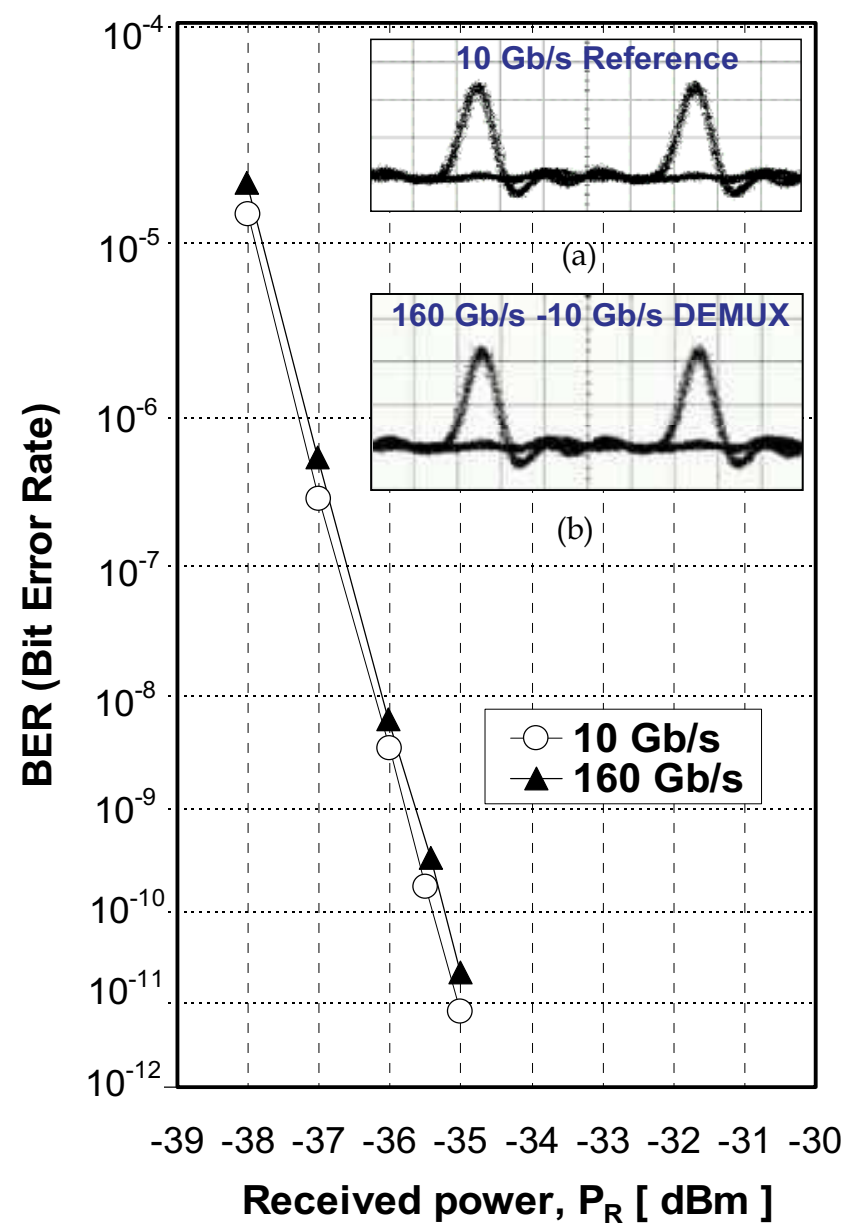

Fig. 10. Bit error rates (BERs) obtained by $160 \mathrm{~Gb} / \mathrm{s}-10 \mathrm{~Gb} / \mathrm{s}$ OTDM DEMUX experiments. The eye-patterns in the BER measurements of $10 \mathrm{~Gb} / \mathrm{s}$ reference (inset (a)) and $160 \mathrm{~Gb} / \mathrm{s}-10$ Gb/s OTDM DEMUX (inset (b)) are also shown.

\section{Conclusion}

In this chapter, we overviewed the developments of highly-nonlinear glass materials and the optical signal processing functions of highly-nonlinear glass-based FOPA. The parametric gain characteristics of highly-nonlinear glass based FOPA in phase-matching conditions were introduced using numerical simulations based on FWM coupling equations. Using the parametric gain characteristics of FOPA in perfect phase-matching condition, in order to realize a practical glass-fiber based FOPA, we proposed the simple and practical design scheme to achieve the broad and large parametric gain characteristics of FOPA using silica glass-based HNLF and single pump As a result, with proposed FOPA design scheme, 
over $20 \mathrm{~dB}$ parametric gain was achieved in $50 \mathrm{~nm}$ band width in the C-band wavelength region. In particular, apparent improvement in the parametric gain characteristics was observed in the wavelength region near the pump wavelength. Furthermore, we performed $160 \mathrm{~Gb} / \mathrm{s}-10 \mathrm{~Gb} / \mathrm{s}$ OTDM DEMUX experiments at the wavelength where the parametric gain characteristics improved. In the ultrafast DEMUX experiments, we verified that no error penalty occurred for $160 \mathrm{~Gb} / \mathrm{s}$ pulse trains. This result shows that our design scheme for FOPA using the perfect phase-matching condition of FWM is effective for the transparent DEMUX of the ultra high bit-rate pulses over $160 \mathrm{~Gb} / \mathrm{s}$.

\section{References}

Agrawal, G. P. (1995). Nonlinear Fiber Optics, 2nd edition, Academic express, San Diego, CA, USA.

Aso, O.; Arai, S.; Yagi, T.; Tadakuma, M.; Suzuki, Y. \& Namiki, S. (2000). Broadband fourwave mixing generation in short optical fibers, Electron. Lett. Vol. 36, pp. 709 - 711.

Asobe, M.; Kanamori, T. \& Kubodera, K. (1993). Applications of highly nonlinear chalcogenide glass fibers in ultrafast all-optical switches, IEEE Journal of Quantum Electronics. Vol. 29, pp. 2325-2333.

Birks, T.A.; Knight, J.C. \& St. J. Russell, P. (1997) Endlessly single-mode photonic crystal fiber, Optics Letters, Vol. 22, No 13, pp. 961-963.

Chow, K. K.; Kikuchi, K.; Nagashima, T.; Hasegawa, T.; Ohara, S. \& Sugimoto, N. (2007). Four-wave mixing based widely tunable wavelength conversion using 1-m dispersion-shifted bismuth-oxide photonic crystal fiber, Optics Express Vol. 15, pp. 15418-15423.

Hedekvist, P. O.; Karlsson, M. \& Andrekson, P. A. (1997). Fiber four-wave mixing demultiplexing with inherent parametric amplification, J. Lightwave Technol., vol.15, pp. $2051-2058$.

Hirano, M.; Nakanishi, T.; Okuno, T. \& Onishi, M. (2005). Broadband Wavelength Conversion over 193-nm by HNL-DSF Improving Higher-order Dispersion Performance, ECOC 2005 Proceedings, Vol. 6, pp. 43-44.

Ho, M. C.; Uesaka, K.; Marhic, M. E.; Akasaka, Y. \& Kazovsky, L. G. (2001). 200-nmbandwidth fiber optical amplifier combining parametric and raman gain, Journal of Lightwave Technology, vol.19, pp. 977 - 981.

Inoue, K. \& Toba, H. (1992). Wavelength conversion experiment using fiber four-wave mixing, IEEE Photonics Technology Letters, Vol. 4, pp. 69-72.

Lee, J. H.; Belardi, W.; Furusawa, K.; Petropoulos, P.; Yusoff, Z.; Monro, T.M.; \& Richardson, D.J. (2003). Four-wave mixing based $10 \mathrm{Gbit} / \mathrm{s}$ tunable wavelength conversion using a holey fiber with a high SBS threshold, IEEE Photonics Technology Letters, Vol.15, pp. 440-442.

Lee, J. H.; Nagashima, T.; Hasegawa, T.; Ohara, S.; Sugimoto, N. \& Kikuchi, K. (2005). Fourwave- mixing-based wavelength conversion of $40-\mathrm{Gb} / \mathrm{s}$ nonreturn-to-zero signal using $40-\mathrm{cm}$ bismuth oxide nonlinear optical fiber, IEEE Photonics Technology Lettes, Vol.17, no.7, pp. 1474-1476.

Li, J.; Hansryd, J.; Hedekvist, P. O.; Andrekson, P. A. \& Knudsen, S. N. (2001). 300Gbit/s eye-diagram measurement by optical sampling using fiber and Exhibit, Optical Fiber Communication Conference, March 17-22, Anaheim, CA. 
Marhic, M. E.; Kagi, N.; Chiang, T.-K. \& Kazovsky, L. G. (1996). Broadband fiber optical parametric amplifiers, Optics Express, Vol. 21, pp. 573-575.

Onishi, M.; Okuno, M.; Kashiwada, T.; Ishikawa, T. ; Akaska, S. \& Nishimura, N. (1997). Integrated Optics and Optical Fiber Communications, 11th International Conference on, and 23rd European Conference on Optical Communications, Vol. 2, pp. $115-118$.

Petropoulos, P.; Ebendorff-Heidepriem,Heike.; Finazzi, V.; Moore, R.; Frampton, K.; Richardson, D. \& Monro, T. (2003). Highly nonlinear and anomalously dispersive lead silicate glass holey fibers, Optics Express Vol.11, pp. 3568-3573.

Yang, F. S.; Ho, M. C.; Marhic, M. E. \& Kazovsky, L. G. (1997). Demonstration of two-pump fiber optical parametric amplification, Lasers and Electro-Optics Society Annual Meeting 1997, LEOS '97 10th Annual Meeting. Vol. 1.

Westlund, M.; Hansryd, J.; Andrekson, P. A. \& Knudsen, S. N. (2002). Trans-parent wavelength conversion in fiber with $24 \mathrm{~nm}$ pump tuning range, Electron. Lett., vol.38, pp. $85-86$. 


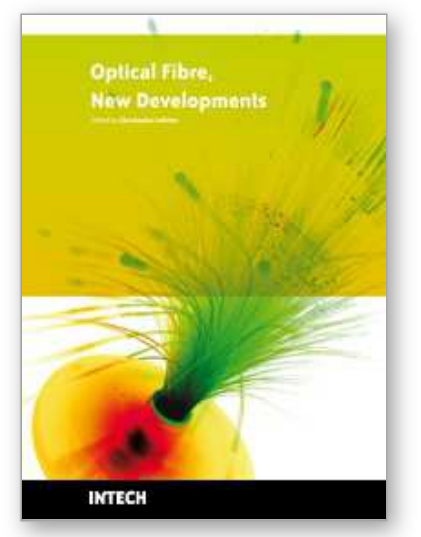

\section{Optical Fiber New Developments}

Edited by Christophe Lethien

ISBN 978-953-7619-50-3

Hard cover, 586 pages

Publisher InTech

Published online 01, December, 2009

Published in print edition December, 2009

The optical fibre technology is one of the hop topics developed in the beginning of the 21 th century and could substantially benefit applications dealing with lighting, sensing and communication systems. Many improvements have been made in the past years to reduce the fibre attenuation and to improve the fibre performance. Nowadays, new applications have been developed over the scientific community and this book fits this paradigm. It summarizes the current status of know-how in optical fibre applications and represents a further source of information dealing with two main topics: the development of fibre optics sensors, and the application of optical fibre for telecommunication systems.

\section{How to reference}

In order to correctly reference this scholarly work, feel free to copy and paste the following:

Shunsuke Ono (2009). Fiber Optical Parametric Amplifier as Optical Signal Processor, Optical Fiber New Developments, Christophe Lethien (Ed.), ISBN: 978-953-7619-50-3, InTech, Available from: http://www.intechopen.com/books/optical-fiber-new-developments/fiber-optical-parametric-amplifier-as-opticalsignal-processor

\section{INTECH}

open science | open minds

\section{InTech Europe}

University Campus STeP Ri

Slavka Krautzeka 83/A

51000 Rijeka, Croatia

Phone: +385 (51) 770447

Fax: +385 (51) 686166

www.intechopen.com

\section{InTech China}

Unit 405, Office Block, Hotel Equatorial Shanghai

No.65, Yan An Road (West), Shanghai, 200040, China

中国上海市延安西路65号上海国际贵都大饭店办公楼405单元

Phone: +86-21-62489820

Fax: $+86-21-62489821$ 
(C) 2009 The Author(s). Licensee IntechOpen. This chapter is distributed under the terms of the Creative Commons Attribution-NonCommercial-ShareAlike-3.0 License, which permits use, distribution and reproduction for non-commercial purposes, provided the original is properly cited and derivative works building on this content are distributed under the same license. 